PREPRINT 2009:26

\title{
Note on the Estimation of Crossing Intensity for Laplace Moving Average
}

\section{THOMAS GALTIER}

Department of Mathematical Sciences Division of Mathematical Statistics

CHALMERS UNIVERSITY OF TECHNOLOGY UNIVERSITY OF GOTHENBURG

Göteborg Sweden 2009 

Preprint 2009:26

\title{
Note on the Estimation of Crossing Intensity for Laplace Moving Average
}

\author{
Thomas Galtier
}

Department of Mathematical Sciences

Division of Mathematical Statistics

Chalmers University of Technology and University of Gothenburg

SE-412 96 Göteborg, Sweden

Göteborg, April 2009 
Preprint 2009:26 ISSN 1652-9715

Matematiska vetenskaper

Göteborg 2009 


\title{
NOTE ON THE ESTIMATION OF CROSSING INTENSITY FOR LAPLACE MOVING AVERAGE
}

\author{
THOMAS GALTIER \\ Mathematical Sciences, \\ Chalmers University of Technology and Göteborg University, \\ 41296 Göteborg, Sweden. \\ E-mail adress: galtier@chalmers.se
}

\begin{abstract}
The intensity of upcrossings of a level u by a Laplace Moving Average (LMA) process is estimated by means of a saddlepoint approximation of Rice's formula. The LMA-process is defined by power spectral density, skewness and kurtosis parameters.

The method is illustrated by measurements of sea elevation and stresses in a vessel.

Key-words: Rice formula; Non-Gaussian process; Non-Gaussian Seas; skewness; kurtosis; saddlepoint approximation; Laplace moving average; mean upcrossing intensity;
\end{abstract}

\section{INTRODUCTION}

In offshore engineering, one of the dominant sources of uncertainties is related to environmental loads. Important characterisation of such loads is the number of times a load upcrosses a level $u$ during the interval of time $[0, T]$, denoted by $N_{T}(u)$. The expected value $E\left[N_{T}(u)\right]$ is an important parameter used in design values and evaluation of safety levels for structures exposed for environmental loads.

Let us denote a load by $Y(t), t \in[0, T]$. In this note we discuss the computation of $E\left[N_{T}(u)\right]$ for Non-Gaussian process $Y(t)$, called Laplace Moving Average, defined in Section 2. We assume that $Y(t)$ is stationary and almost surely continuously differentiable, then $E\left[N_{T}(u)\right]=T \mu(u)$, where $\mu(u)$ is the intensity of $u$-upcrossings given by the celebrated Rice's formula:

Theorem 1. If the process $Y(t)$ is a.s. differentiable, then the expected number of times the process $Y(t)$ crosses $u$ in the upward direction $\mu(u)$ is 
given by

$$
\mu(u) \stackrel{\text { a.a.u }}{=} \int_{0}^{+\infty} z f_{Y(0), \dot{Y}(0)}(u, z) d z
$$

where $f_{Y(0), \dot{Y}(0)}(u, z)$ is the joint density of $Y(0), \dot{Y}(0)$ and a.a.u shows that the formula is always true for almost all values of $u$.

For proof of this theorem, see Brillinger [10] or Zahle [11]. Under more restrictive assumptions, see Marcus [7], Rice's formula (1.1) is true for a fixed level $u$.

Examples of relevant applications of $\mu(u)$ are:

- To bound the distribution of the maximal load (highest wave crest or maximum response), $M_{T}=\max _{\{0 \leq t \leq T\}} Y(t)$ - where $Y(t)$ is the sea surface elevation - during a specified interval of time $T$. The distribution of $M_{T}$ can be bounded as follows

$$
P\left(M_{T}>u\right) \leq P(Y(0)>u)+E\left[N_{T}(u)\right] .
$$

- To model the ageing process in materials (fatigue analysis); as damage accumulates with a rate depending both on the frequency and the magnitude of the sea waves. As shown in Rychlik [9] the damage rate $d$ can be bounded by

$$
d \leq c \int_{0}^{+\infty} u^{\beta} \mu(u) d u
$$

with equality when $u$ tends to infinity. Here constants $c, \beta$ are suitable dependent material.

- To define a transformed Gaussian process which is then used to model sea waves; see Johannesson et al. [12] for more details.

The Gaussian processes are extensively studied and many results (tools) are available. For example, if $Y(t)$ is a zero mean Gaussian process, (1.1) is an explicit form, viz.

$$
\mu(u)=\frac{1}{2} \sqrt{\frac{\operatorname{Var}[\dot{Y}(0)]}{\operatorname{Var}[Y(0)]}} \exp \left(-\frac{u^{2}}{2 \operatorname{Var}[Y(0)]}\right) .
$$

However, in many cases the departure from Gaussianity cannot be neglected. In this article we will focus on wave loads, see in Section 4. In the literature, Stokes waves are often used to describe the non-linear behaviour of waves. These are defined by means of a spectrum and some physical modelling. In this approach the sea surface is expressed as a second-order Volterra series of Gaussian processes. Here we will use the LMA-process to model the wave loads. The LMA-process will have both a "correct" correlation structure, skewness and kurtosis. However, contrary to Gaussian processes, now (1.1) cannot be computed in an explicit form. In the literature, one 
can find different methods and tools to estimate Rice's formula in the case of a second-order Volterra series (Stokes waves), see Naess [5], Machado [4], or Breitung [13] and the asymptotic expansion method of Hagberg [8]. In this article we will in particular focus on the saddlepoint approximation proposed in Machado et al. [3] and adapt it to an LMA case. The method uses the cumulant generating function, see Section 3. The accuracy of the proposed approach is shown in Section 4.

\section{Laplace Moving Average}

A short overview of the Generalized Laplace Laws and some essential properties of the Laplace moving average will be given here, see Kotz et al. [2] for more details on this subject. Following [2], the generalized Laplace laws are described by their characteristic functions, and, in the one dimensional case, are given by

$$
\phi(u)=\left(1-i \mu u+\frac{\sigma^{2} u^{2}}{2}\right)^{1 / \nu}
$$

where $\mu \in R$ and $\sigma>0$. We use $\mathcal{L}(\mu, \sigma, \nu)$ for the above-defined distribution with the standard values of the parameters: $\mu=0$ (symmetric case), scale $\sigma=1$, and shape $\nu=1$. By default, if any of the parameters are dropped from the notation they are assumed to be set to their standard value.
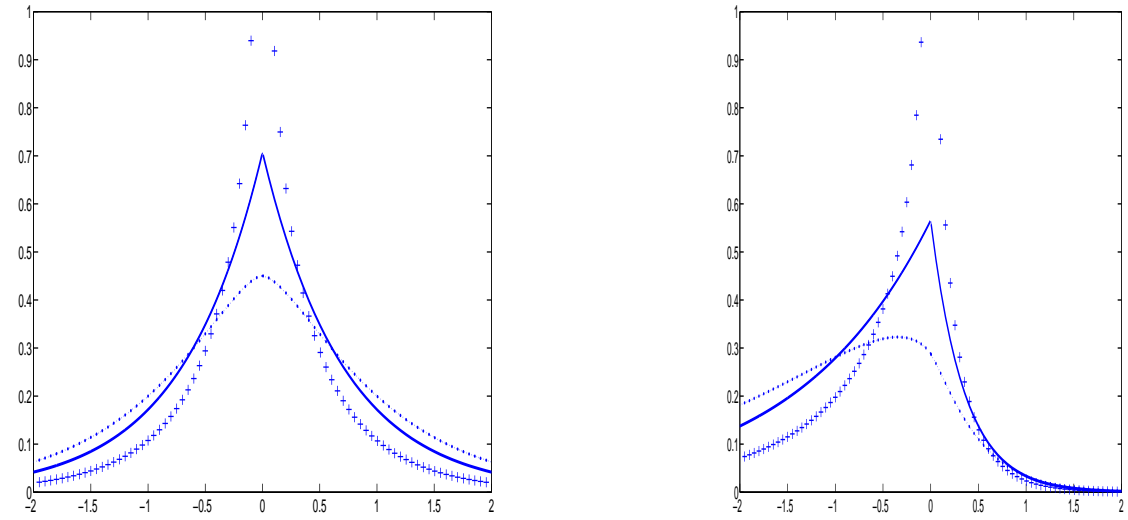

FIGURE 1. Symmetric (left) and asymmetric (right) Laplace distribution with scale parameter $\sigma=1$, and shape parameter $1 / \nu=\frac{1}{2}$ (crossed line), $1 / \nu=1$ (solid line), $1 / \nu=\frac{3}{2}$ (dotted line), respectively. 
Definition 1. (LAPLACE MOTION). A Laplace Motion $L(t)$ with the asymmetry parameter $\mu$, the space scale parameter $\sigma$ and the time scale parameter $\nu, \mathcal{L} \mathcal{M}(\mu, \sigma, \nu)$ is defined by the following conditions

(1) it starts at the origin, i.e., $L(0)=0$;

(2) it has independent and stationary increments;

(3) the increments by the time scale unit have a symmetric Laplace distribution with the parameter $\sigma$, i.e.,

$$
L(t+\nu)-L(t) \stackrel{d}{=} \mathcal{L}(\mu, \sigma)
$$
motion.

If $\mu=0, \sigma=1$ and $\nu=1$ the process $L(t)$ is called standard Laplace

Following [2] we can define the process referred as a Laplace Moving Average by

$$
Y(t)=\int_{\mathbb{R}} f(t-x) d \Lambda(x) .
$$

Since the scaling of the kernel function $f$ can be equivalently expressed by the corresponding scaling parameter $\nu$, we always assume that $f$ is scaled so that $\int f^{2}=1$. The next result lists basic facts about this class of second-order processes. Here and in what follows, the Fourier transform is defined by

$$
\mathcal{F}\{f\}(\omega)=\int_{\mathbb{R}} \exp (-i \omega t) f(t) d t .
$$

Theorem 2. Let $\Lambda$ be a stochastic Laplace measure with parameters $\mu$ and $\sigma$ controlled by the Lebesgue measure on $\mathbb{R}$ that is divided by $\nu$. Further, let $Y(t)$ be the moving average process defined by (2.1). Then

(1) the marginal distribution of $Y(t)$ is given by the characteristic function

$$
\begin{aligned}
& \phi_{Y(t)}(u)=\exp \left(-\frac{1}{\nu} \int_{\mathbb{R}} \log \left(1-i \mu u f(x)+\frac{\sigma^{2} f^{2}(x) u^{2}}{2}\right) d x\right) \\
& \text { and more generally its finite dimensional distribution of } \\
& Y(t)=\left(Y\left(t_{1}\right), \ldots, Y\left(t_{n}\right)\right) \text { is given by the characteristic function } \\
& \text { at } \boldsymbol{u}=\left(u_{1}, \ldots, u_{n}\right) \\
& \phi_{Y(\boldsymbol{t})}(\boldsymbol{u})=\exp \left(-\frac{1}{\nu} \int_{\mathbb{R}^{n}} \log \left(1-i \mu \boldsymbol{u}^{T} f_{\boldsymbol{t}}(x)+\frac{\sigma^{2}}{2}\left(\boldsymbol{u}^{T} f_{\boldsymbol{t}}(x) f_{\boldsymbol{t}}^{T}(x) \boldsymbol{u}\right)\right) d x\right) \\
& \text { where } f_{\boldsymbol{t}}(x)=\left(f_{t_{1}}(x), \ldots, f_{t_{n}}(x)\right) \text { is the vector of the kernel func- } \\
& \text { tion. }
\end{aligned}
$$


(2) The autocorrelation function $\rho(\tau)$ of $Y(t)$ is given by

$$
\rho(\tau)=\int_{-\infty}^{+\infty} f(x-\tau) f(x) d x=(f * \tilde{f})(\tau),
$$

where $\tilde{f}(x)=f(-x)$ and $*$ denotes the convolution operator.

(3) In $\mathbb{R}$, the spectral density $R(\omega)$ of $Y(t)$ is given by

$$
R(\omega)=\frac{\sigma^{2}+\mu^{2}}{\nu} \cdot \frac{\mathcal{F}\{f(\omega)\} \mathcal{F}\{\tilde{f}(\omega)\}}{2 \pi}
$$

In particular, if $f$ is symmetric, then

$$
R(\omega)=\frac{\sigma^{2}+\mu^{2}}{\nu}\left|\mathcal{F}\{f(\omega)\}^{2}\right| /(2 \pi) .
$$

\section{SAddepoint Method}

If the kernel function is differentiable then the derivative of the process $\dot{Y}(t)$ exists and the expected number of times the process $Y(t)$ crosses $u$ in the upward direction $\mu(u)$, is given by the Rice formula (1.1). Daniels [6] introduces the saddlepoint approximation as a formula to approximate the probability density function from the cumulant generating function. In Machado et al. [3] the method is used to derive an approximation of $\mu(u)$. As mentioned before, the saddlepoint method is for using the cumulant generating function

$$
\begin{aligned}
K(s, t) & =\ln \left\{E\left[e^{-i s Y(0)-i t \dot{Y}(0)}\right]\right\} \\
& =\ln \left\{\phi_{Y(0), \dot{Y}(0)}(-i s,-i t)\right\},
\end{aligned}
$$

where $\phi_{Y(0), \dot{Y}(0)}$ is the joint characteristic function of $Y(0)$ and $\dot{Y}(0)$.

Following [3], for the symmetrical cumulant generating function $K(s, t)$ in $t$, i.e. $K(s, t)=K(s,-t)$, one has the following expression for the approximation of the mean upcrossing intensity $\mu(u)$

$$
\mu(u) \approx \hat{f}(u) \frac{\sqrt{g^{\prime \prime}(0)}}{\sqrt{2 \pi}}\left(1+\frac{h^{\prime \prime}(0)}{2 h(0) g^{\prime \prime}(0)}-\frac{1}{24} \frac{g^{i v}(0)}{g^{\prime \prime}(0)^{2}}\right)=\mu^{s d}(u)
$$

where $g(t)=K\left(s_{t}, t\right)-s_{t} u, h(t)=\frac{1}{\sqrt{K_{11}\left(s_{t}, t\right)}}$ and $s_{t}$ satisfies $K_{1}\left(s_{t}, t\right)=$ $u$.

Here

$$
K_{1}(s, t)=\frac{\partial K(s, t)}{\partial s}, \quad K_{11}(s, t)=\frac{\partial^{2} K(s, t)}{\partial s^{2}}
$$

and $\hat{f}(u)$ is the saddlepoint approximation for the density of $Y(0)$ given by 


$$
\hat{f}(u)=\frac{h(0)}{\sqrt{2 \pi}} e^{g(0)}
$$

Consider $Y(t)$ as an LMA-process defined as in (2.1). Since

$$
s Y(0)+t \dot{Y}(0)=\int_{-\infty}^{+\infty}(s f(x)+t \dot{f}(x)) d \Lambda(x)
$$

By (1), in Theorem 2, we show that

$\phi_{Y(0), \dot{Y}(0)}(s, t)=\exp \left(i \gamma \int_{-\infty}^{+\infty} s f(x)+t \dot{f}(x) d x\right) \cdot \exp \left(-\frac{1}{\nu} \int_{-\infty}^{+\infty} \log (r(x ; s, t)) d x\right)$

where $r(x ; s, t)=1-i \mu(s f(x)+t \dot{f}(x))+\frac{\sigma^{2}}{2}(s f(x)+t \dot{f}(x))^{2}$.

Hence the cumulant generating function is given by

$$
K(s, t)=\gamma \int_{-\infty}^{+\infty} s f(x)+t \dot{f}(x) d x-\frac{1}{\nu} \int_{-\infty}^{+\infty} \log (r(x ; s, t)) d x
$$

It is easy to show that $K(s, t)=K(s,-t)$ and hence one can apply (3.1) to approximate $\mu(u)$.

\section{NUMERICAL EXAMPLES}

In this section we will illustrate the accuracy of the saddlepoint approximation of the upcrossing intensity $\mu(u)$ for LMA-processes. Two typical signals for offshore engineering will be considered; the sea surface elevation measured at a fixed location and the stresses in a vessel.

The two signals will be modelled by means of Gaussian and LMA processes. For two models, the intensity of the upcrossings will be computed and will be compared with the observed upcrossings in the signals.

Note that for a zero-mean stationary Gaussian process the number of times the process crosses the level $u$ in the upward direction per time units is given by the algebraic form (1.2).

4.1. Measured sea level. Let consider forty minutes measurements of sea surface elevation at a fixed location (see Figure 2 left plot). This is a slightly non Gaussian signal having kurtosis equal to 3.1732 and skewness equal to 0.2546 , which are typical values for this type of signals. Often Gaussian process would be used to describe the signal. 

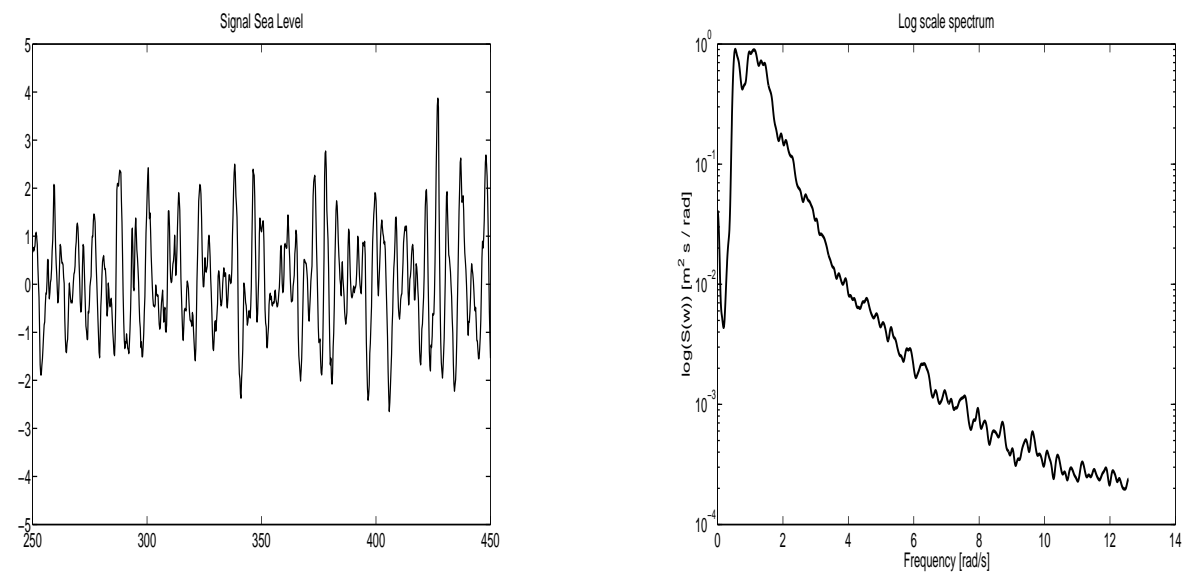

FIGURE 2. Measured sea surface elevation (left) and corresponding power spectral density (p.s.d.) in log scale (right).

Let us assume $Y(t)$ as being an LMA. The process $Y(t)$ is defined by mean, variance, skewness, kurtosis and the kernel function $f(t)$ (see Figure 3 , left plot). Note that $f(t)$ is not uniquely defined by p.s.d. We choose the symmetrical and normed kernel given by (2.2). Parameters of the Laplace Motion, see Definition 1, are computed following Åberg et al. [1], using observed values of variance, skewness and kurtosis.

In Figure 3 (right plot), the observed intensity of the upcrossings (irregular solid line) is compared with the theoretical intensity of the upcrossings computed for the Gaussian process (dashed line) and saddlepoint approximation $\mu^{s d}(u)$ of the intensity of th upcrossings for the LMA.
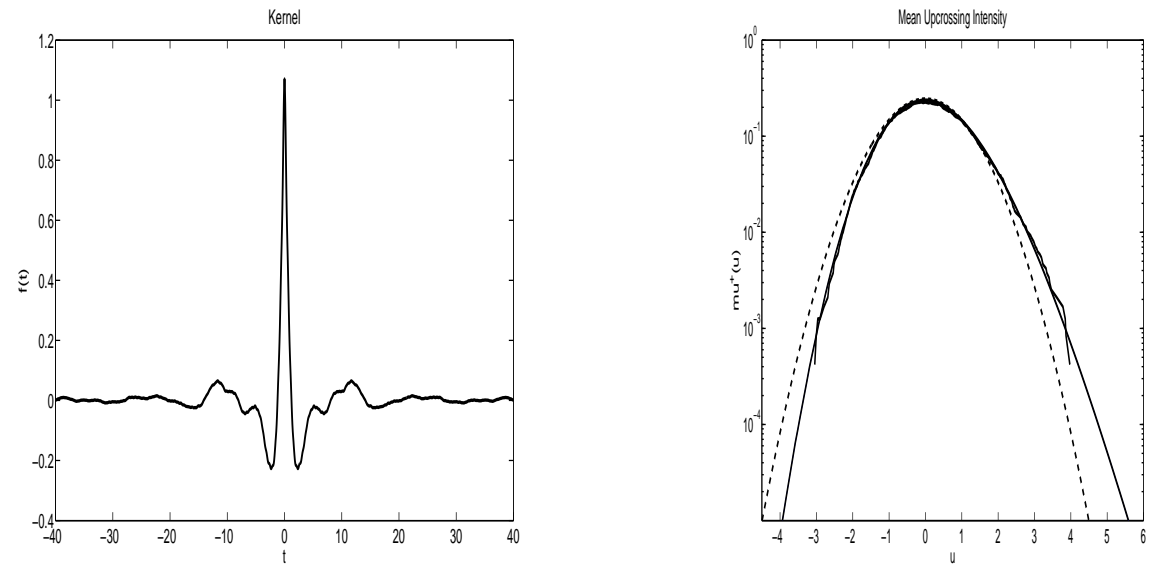

FIGURE 3. Kernel function $f(t)$ derived from p.s.d in Figure 2 right plot (left). Mean upcrossing intensity for sea level (right); Observed signal (irregular solid line), saddlepoint approximation (regular solid line) and from Rice formula (1.2) under Gaussian assumption (dashed line). 
We see that the observed intensity of the upcrossings and $\mu^{s d}(u)$ are very close. The intensity of the upcrossings computed for the Gaussian process is less accurate.

4.2. Measured Stresses. The second example is the thirty-minute measured stresses in the after section of a container ship (see Figure 4, left plot). Here the kurtosis is equal to 7.6572 and the skewness is equal to 1.1219. Obviously, this signal is not a realisation of a Gaussian process. Again, the LMA process will be used to model the variability of the signal. Similarly as in Section 4.1, we estimated the four parameters of the LMA and computed the kernel function (see Figure 5, left plot) as in (2.2). In Figure 4 (right plot), we can see that the power spectral density has more peaks than the p.s.d. shown in Figure 2. These are caused by resonances of the structure.
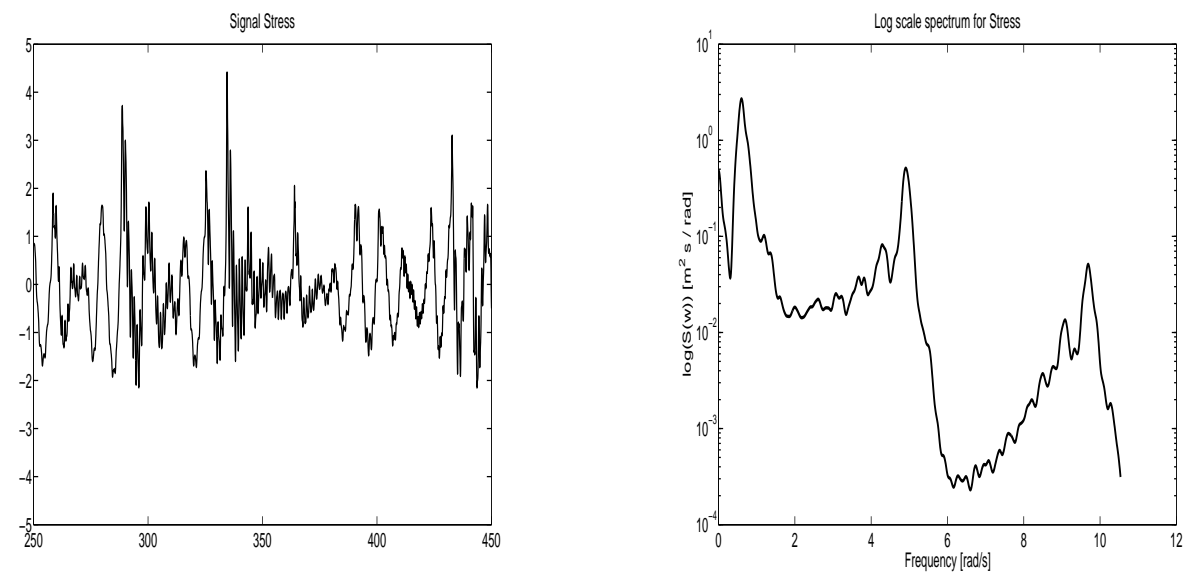

FIGURE 4. Measured stress in a container ship (left) and corresponding power spectral density (p.s.d.) in log scale (right).

In Figure 5 (right plot), the irregular solid line is the observed intensity of the upcrossings and the regular line is $\mu^{s d}(u)$ ). Again, the agreement between the observed intensity of the upcrossings and $\mu^{s d}(u)$ is excellent. For comparison, upcrossings for a Gaussian process having the p.s.d. is shown in Figure 5, also as a dashed line. 

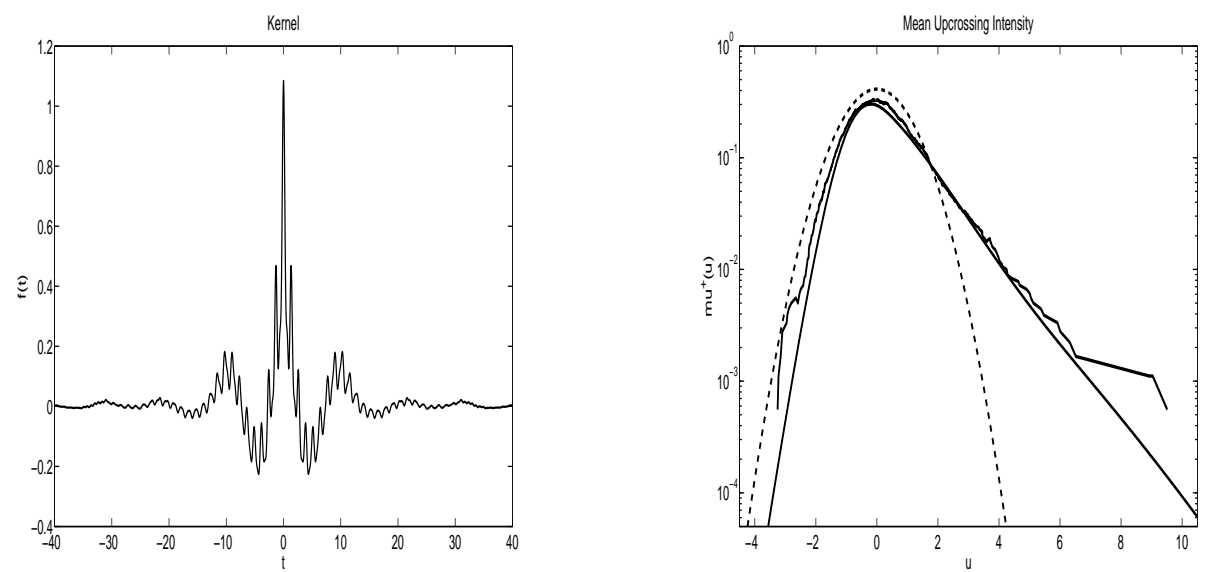

FIGURE 5. Kernel function $f(t)$ derived from p.s.d. in figure 4, right plot (left). Mean upcrossing intensity for structural response (right); Observed signal (irregular solid line), saddlepoint approximation (regular solid line) and from Rice formula (1.2) under Gaussian assumption (dashed line).

We can see that the Gaussian model cannot be used here.

\section{Conclusion}

In this paper we have shown that the intensity of the upcrossings of Non-Gaussian signals could be accurately estimated by means of the theoretical intensity of the upcrossings of LMA models. We have also demonstrated that the saddlepoint method can be used to approximate $\mu(u)$ for high values of $u$.

\section{ACKNOWLEDgement}

This research was supported in part by Gothenburg Stochastic Center and the Swedish foundation for strategic research through GMMC, Gothenburg Mathematical Modelling Center and in part by the E.U. project SEAMOCS. The author is grateful to Sofia Åberg for discussions. The author is also grateful to Gaute Storhaug from Det Norske Veritas (DnV) in Hövik, for the data presented in Section 4.2.

\section{REFERENCES}

[1] Åberg, S., Podgórski, K., RYChlik, I.: Fatigue damage assessment for a spectral model of non-Gaussian random loads, Preprints in Mathematical Sciences, to appear in Probabilistic Engineering Mechanics, (2009).

[2] Kotz, S., Kozubowski, T.J., PodgóRski, K.: The Laplace distribution and generalizations: A revisit with applications to communications, economics, engineering and finance, Birkhaüser, Boston,(2001). 
[3] Machado, U.B., Butler, R.W., Rychlik, I.: Distribution of Wave Crests in Non-Gaussian Sea, To appear in Applied Ocean Research, (2009).

[4] Machado, U.B.: Statistical Analysis of Non-Gaussian Environmental Loads and Responses, Lund University, Sweden, (2002), Phd Thesis ISBN 91-628-5254-X, 190 pp.

[5] NAESS,A., GAIDAI,O.: The asymptotic behaviour of second-order stochastic Volterra series models of slow drift response, Probabilistic Engineering Mechanics 22, (2007), 343-352.

[6] Daniels, H.E.: Saddlepoint approximations in statistics, Ann. Math. Statis., 25, (1954), 631-50.

[7] MARCUS, M.B.: Level crossings of a stochastic process with absolutely continuous sample paths, The Annals of Probability , (1977), 5, 52-71.

[8] HagBerg, O.: Asymptotic expansions of crossing rates of stationary random processes, Lund University, Sweden, (2005), Phd Thesis ISBN 91-628-6384-3, 129 pp.

[9] RYCHLIK, I.: On the narrow-band approximation for expected fatigue damage, Probabilistic Engineering Mechanics, (1993), 8, 1-4.

[10] BRILlingeR, D.R.: On the number of solutions of systems of random Equations, Ann. Math. Statist. , (1972), 43, 534-540.

[11] ZÄHLE, U.: A general Rice formula, Palm measures, and horizontal window conditioning for random fields, Stochastic Process and Their Applications, (1984), 17, 265-283.

[12] Johannesson, P., Rychlik, I., Leadbetter, M.R.: Modelling and Statistical Analysis of Ocean-Data Using Transform Gaussian Processes, Marine Structures, (1997), 10, 13-47.

[13] BREITUNG, K.: Asymptotic crossing rates for stationary Gaussian vector processes, Stochastic Process and Their Applications, (1988), 29, 195-207. 